\title{
MODEL OF HARDENING ELEMENTS OF TOOLS STEEL C80U
}

\author{
Tomasz Domański \\ Institute of Mechanics and Machine Design, Czestochowa University of Technology \\ Czestochowa, Poland \\ domanski@imipkm.pcz.pl
}

\begin{abstract}
Hardening is one of the manufacturing processes used for improving mechanical properties such as strength, hardness and wear resistances of the mechanical components for machine parts. This paper describes modelling of the processes of steel C80U hardening. The first priority was given to thermal phenomena, phase transformations in a solid state and mechanical phenomena. A numerical algorithm of thermal phenomena was based on the Finite Elements Methods in the Galerkin formula of the heat transfer equations. In the model of phase transformations, in simulations heating process, isothermal or continuous heating (CHT) was applied, whereas in the cooling process continuous cooling (CCT) of the steel. In the model of mechanical phenomena, apart from thermal, plastic and structural strain, transformations plasticity was also taken into account. The stress and strain fields are obtained using the solution of the Finite Elements Method of the equilibrium equation in rate form. The thermophysical constants occurring in constitutive relation depend on temperature and phase composite. In order to determine plastic strain, the Huber-Mises condition with isotropic strengthening was applied, whereas for determination of transformation plasticity a modified Leblond model was used. In order to evaluate the quality and usefulness of the presented models a numerical analysis of temperature field, phase fraction, stress and strain associated hardening process of a cone-shaped fang lathe made of tool steel was carried out.
\end{abstract}

Keywords: heat treatment, phase transformations, stress, strain, numerical modelling

\section{Introduction}

Thermal treatment including hardening is a complex technological process aimed at obtaining high hardness, high abrasion resistance, high durability of the elements hardened as well as suitable initial structure to be used in the subsequent thermal treatment processes as a result of which the optimum mechanical properties of the elements are received. The product of the martensite transformation is the primary structure of the steel undergoing hardening.

Today an intense development of numerical methods supporting designing or improvement of already existing technological processes is observed. The technologies mentioned above also include steel thermal processing comprising hardening. Efforts involving thermal processing of numerical models aim to encompass an increasing number of input parameters of such a process. 
The prediction of final properties of the element undergoing hardening is possible after determination of the type and features of the microstructure to be created, as well as of instantaneous and specific stresses accompanying such technology of product quality improvement. For this to be achieved, it is necessary to take into account, first of all, thermal phenomena, phase transitions and mechanical circumstances in the numerical model.

The Finite Element Method is the method most frequently used to implement numerical algorithms. This method enables one to easily include in the analysis both non-linearity and non-homogeneity of the material thermally processed and therefore in the proposed models both heat conduction equation and energy balance equation are solved using the Finite Element Method [1].

Accuracy of the proposed tool steel hardening methods was proved by comparing the results of numerical simulations and experimental research results presented in the studies [2-4].

\section{Problem formulations, temperature fields, stress and strain}

Temperature fields are obtained with solved of heat transfer equation with source unit:

$$
\nabla \cdot(\lambda \nabla T)-C \frac{\partial T}{\partial t}=-Q
$$

Equation (1) was completed with initial conditions:

$$
T\left(x_{\alpha}, t_{0}\right)=T_{0}\left(x_{\alpha}\right), \quad Q\left(x_{\alpha}, t_{0}\right)=Q\left(x_{\alpha}\right)=0
$$

Heating and cooling can be modeled using the Neumann boundary condition (heating heat flux $q_{n}$ on $\Gamma_{q}$ ) or the Newton boundary (heat flux $\left(q_{n}\right)$ on $\Gamma_{\infty}$ depends on the temperature of boundary elements and surrounding medium temperature), i.e.:

$$
\begin{aligned}
& q_{n}=-\left.\lambda \frac{\partial T}{\partial n}\right|_{\Gamma_{q}}=q^{*}\left(\left.x_{\alpha}\right|_{\Gamma_{q}}, t\right) \\
& q_{n}=-\left.\lambda \frac{\partial T}{\partial n}\right|_{\Gamma_{\infty}}=\alpha_{\infty}(T)\left(\left.T\right|_{\Gamma_{\infty}}-T_{\infty}\right)
\end{aligned}
$$

In the model of mechanical phenomena the equations of equilibrium and constitutive relationship are accepted in the rate form $[5,6]$ :

$$
\nabla \dot{\boldsymbol{\sigma}}\left(x_{\alpha}, t\right)=\mathbf{0}, \quad \dot{\boldsymbol{\sigma}}=\dot{\boldsymbol{\sigma}}^{T}, \dot{\boldsymbol{\sigma}}=\mathbf{D} \circ \dot{\boldsymbol{\varepsilon}}^{e}+\dot{\mathbf{D}} \circ \boldsymbol{\varepsilon}^{e}
$$


where: $\boldsymbol{\sigma}=\boldsymbol{\sigma}\left(\sigma_{\alpha \beta}\right)$ is stress tensor, $\mathbf{D}=\mathbf{D}(v, E)$ is tensor of material constant (isotropic material), $v$ is Poisson coefficient, $E=E(T)$ is Young's modulus dependent on temperature, whereas $\boldsymbol{\varepsilon}^{e}$ is the tensor of elastic strain.

Make an assumption the additive of strains, total strain in environment of considered point are results a sum:

$$
\boldsymbol{\varepsilon}=\boldsymbol{\varepsilon}^{e}+\boldsymbol{\varepsilon}^{T p h}+\boldsymbol{\varepsilon}^{t p}+\boldsymbol{\varepsilon}^{p}
$$

where: $\boldsymbol{\varepsilon}^{T p h}$ are isotropic temperature and structural strain, $\boldsymbol{\varepsilon}^{t p}$ are transformations plasticity, whereas $\boldsymbol{\varepsilon}^{p}$ are plastic strain.

\section{Example of hardening elements}

In the simulations of hardening subject was the fang lathe of a cone (axisymmetrical object) made of tool steel. The shape and dimensions of the considered object were presented in Figure 1.

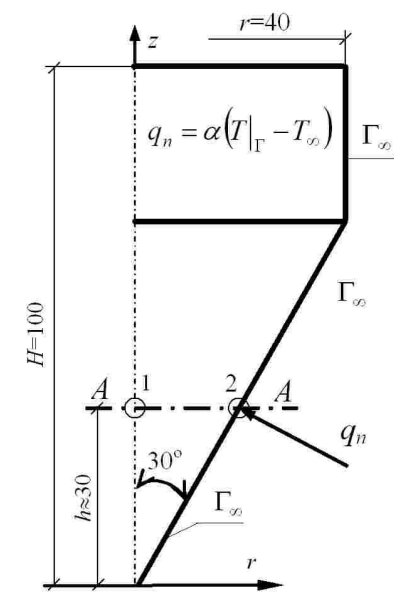

Fig. 1. Scheme and dimensions of hardening object

The superficial heating (surface hardening) of the section of side surface of a cone was modelling the Neumann boundary conditions taken by Gauss distributions of heating source:

$$
q_{n}=\frac{Q}{2 \pi r^{2}} \exp \left(-\frac{(z-h)^{2}}{2 r^{2} \cos \alpha^{2}}\right)
$$

The peak value of the heating source is established on $Q=3500 \mathrm{~W}$, radius $r=15 \mathrm{~mm}$, angle $\alpha=30^{\circ}$ (Fig. 1). The cooling of boundary contact with air was 
modelled by boundary conditions taking $\alpha_{0}=30 \mathrm{~W} /\left(\mathrm{m}^{2} \mathrm{~K}\right)$ [4]. The initial structure was pearlite. The thermophysical values occurrence in conductivity equations $(\lambda, C)$ were taken constant, as the average values from passed in work data $[5,7]$ suitable assumed: $35 \mathrm{~W} /(\mathrm{mK}), 644 * 7760 \approx 5.0 \cdot 10^{6} \mathrm{~J} /\left(\mathrm{m}^{3} \mathrm{~K}\right)$. The heat of phase transformations was determined in work [5] assumed: $\mathrm{H}_{\mathrm{A}-\mathrm{P}}=800 \cdot 10^{6}, \mathrm{H}_{\mathrm{A}-\mathrm{B}}=314 \cdot 10^{6}$, $\mathrm{H}_{\mathrm{A}-\mathrm{M}}=630 \cdot 10^{6} \mathrm{~J} /\left(\mathrm{m}^{3} \mathrm{~K}\right)$. The initial temperature and ambient temperature was assumed equal to $300 \mathrm{~K}$.

In the modelling of mechanical phenomena the Young's and tangential modulus ( $E$ and $E^{t}$ ) was dependent on temperature, however the yield point $\left(Y_{0}\right)$ was dependent on temperature and phase fractions. The values approximated of square functions assumed: Young's and tangential modulus $2.2 \cdot 10^{5}$ and $1.1 \cdot 10^{4} \mathrm{MPa}$ $\left(E_{t}=0.05 E\right)$, yield points $150,400,800$ and $270 \mathrm{MPa}$ suitably for austenite, bainite, martensite and pearlite, in temperature $300 \mathrm{~K}$. In temperature $1700 \mathrm{~K}$ Young's and tangential modulus average 100 and $5 \mathrm{MPa}$ suitable, however yield points are equal to $5 \mathrm{MPa}$. Young's and tangential modulus and yield point for pearlite in temperature $300 \mathrm{~K}$ is established on the basis of its own research estimated on an experimental tension graph for considered steel. The other values are assumed in the literature. The yield point for martensite assumed as average values presented through authors of works $[3,8]$. The heating performed to the moment of cross maximal temperature $1500 \mathrm{~K}$ in environment of a heat source. This ensured obtain the desired phase of austenite in the conical part of fang lathe.

The cooling simulated by flux results from the difference of temperature among the side surface and cooling medium (Newton condition). The temperature of cooling medium is equal to $300 \mathrm{~K}$. The coefficient of thermal conductivity was constant and was equal to $\alpha^{T}=4000 \mathrm{~W} /\left(\mathrm{m}^{2} \mathrm{~K}\right)$ (cooling in fluid layer). Cooling was carried out to obtain the ambient temperature at the boundary of the object and then affixed the final was determined of residual stresses.

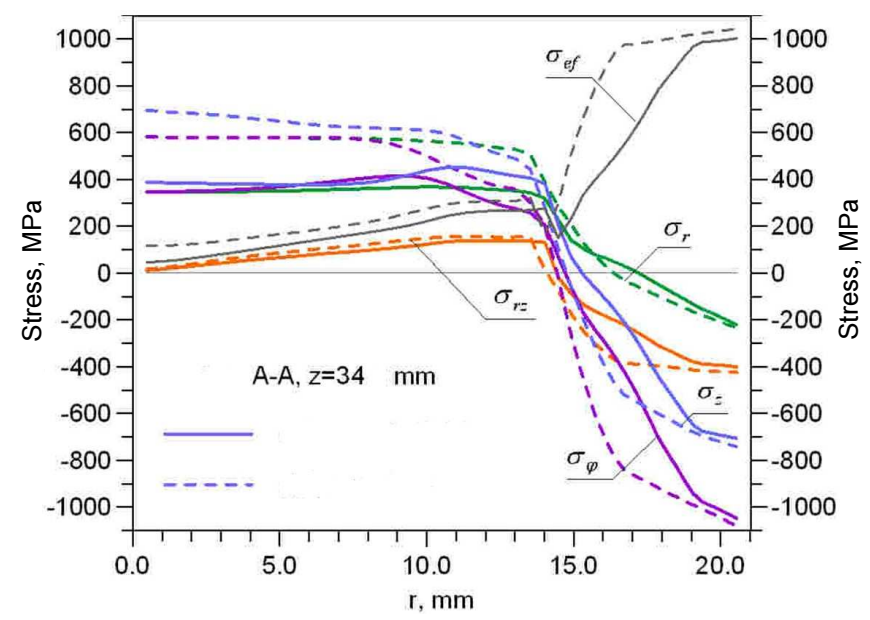

Fig. 2. Stress distributions along the radius 

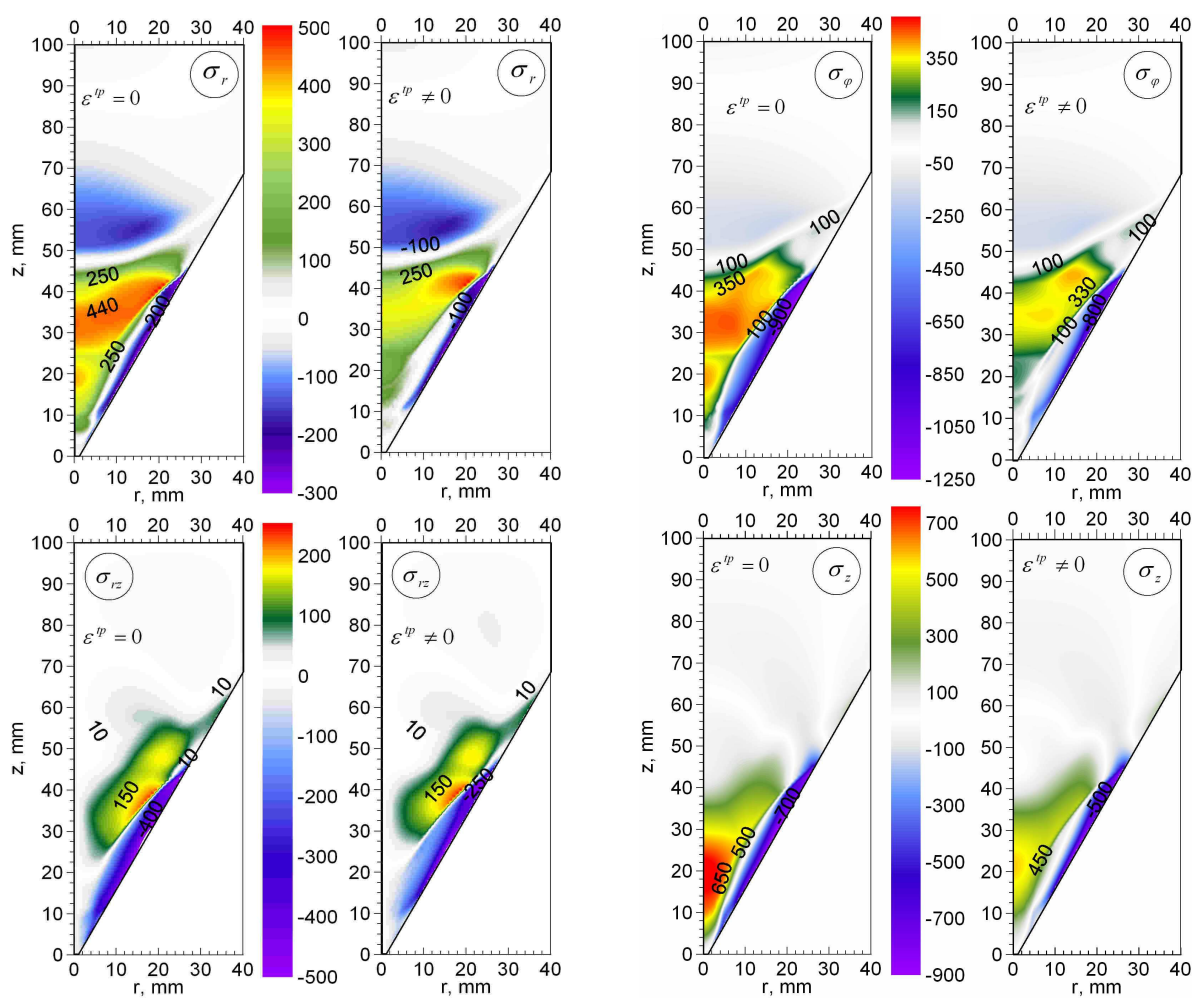

Fig. 3. Distributions of residual stresses (radial, circumferential, tangential, axial) with and without transformations plasticity

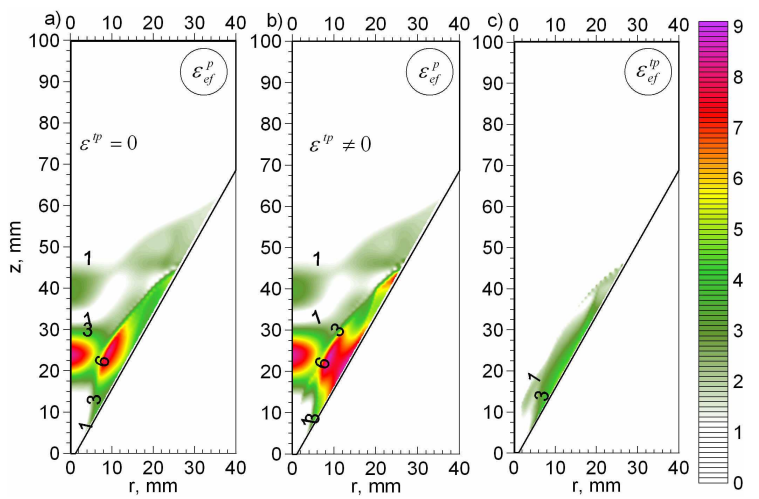

Fig. 4. Distributions of effective plastic strain and transformations plasticity $\left(\cdot 10^{3}\right)$ :

a) without transformations plasticity, b) with transformations plasticity,

c) transformations plasticity

Stress and effective strain distributions obtained in the simulation with and without taking into account transformation plasticity was presented in the figures below. It turns out that the effect of the strain to generate the stress is small in the case of that type of hardening. 


\section{Conclusions}

Distribution of stresses after such hardening is beneficial, as well. The accumulation of stresses is observed only in the zone undergoing hardening, and normal stresses are negative in the subsurface layer (Fig. 2). There are almost no stresses in the lathe core and point. Influence of transformation plasticity is noticeable (see Fig. 3) but is insignificant since it is subsurface hardening. However, taking structural strain into account is very significant for mechanical phenomena. This is presented in the figure displaying the history of instantaneous stresses in the highlighted points of the section A-A. The plastic strain zone is beneficial since it was created in the working part of the lathe. Yet, plastic and transformation strain are not high (Fig. 4). The increased yield point received in the hardened zone (working part of the lathe) is also valuable. It indicates increased hardness of the subsurface layers of this part of the heavy-duty fang lathe undergoing hardening.

\section{References}

[1] Majchrzak E., Mochnacki B., Suchy J., Numerical simulation of thermal processes proceeding in a multi-layered film subjected to ultrafast laser heating, Journal of Theoretical and Applied Mechanics 2009, 47, 2, 385-396.

[2] Ferguson B.L., Li Z., Freborg A.M., Modeling heat treatment of steel parts, Computational Materials Science 2005, 34, 274-281.

[3] Kang S.H., Im Y.T., Thermo-elasto-plastic finite element analysis of quenching process of carbon steel, Journal of Materials Processing Technology 2007, 192-193, 381-390.

[4] Cheng H., Xie J., Li J., Determination of surface heat-transfer coefficients of steel cylinder with phase transformation during gas quenching with high pressures, Computational Materials Science 2004, 29, 453-458.

[5] Cherkaoui M., Berveiller M., Sabar H., Micromechanical modeling of martensitic transformation induced plasticity (TRIP) in austenitic single crystals, International Journal of Plasticity 1998, 14, 7, 597-626.

[6] Bokota A., Domański T., Modelling and numerical analysis of hardening phenomena of tools steel elements, Archives of Metallurgy and Materials 2009, 3, 54, 499-511.

[7] Bokota A., Domański T., Numerical analysis of thermo-mechanical phenomena of hardening process of elements made of carbon steel C80U, Archives of Metallurgy and Materials 2007, 2, $52,277-288$

[8] Leblond J.B., Devaux J., A new kinetic model for anisothermal metallurgical transformation in steels including effect of austenite grain size, Acta Metallurgica 1984, 52, 137-146. 